\title{
SIMULATION METAMODELLING OF MANUFACTURING PROCESSES
}

This paper deals with models going out of simulation models, so called metamodels. They represent one of the possible ways how to increase effectiveness of simulation optimization, how to reduce the requirements of time-consuming simulation of manufacturing systems and how to make simulation cheaper. The heart of the paper describes steps of a metamodel making process and approximation of a real data using regression analysis and least square method.

Keywords: simulation, metamodelling

\section{Introduction}

Rapidly changing market environment and constantly new and higher requirements of demanding customers bring frequent type or volume product changes to the production. The main pressure from customers' side that manufacturer has to manage is the pressure for reducing costs, increasing efficiency and quality which is already commonplace nowadays. It is not possible to handle it, and simultaneously to touch competitive, without continuous improvement of factory processes, implementation of progressive management approaches, technologies, and without rapid decision-making of managers.

Currently, it is axiomatic to solve complex problems by an appropriate computer model that reflects characteristics of a real system or helps to find a solution close to optimal, or directly optimal, for existing or conceptual systems. Therefore, a computer simulation is still gaining major importance. It allows quick testing of various variants of solutions and it minimizes the risk of wrong decisions. This is reflected to considerable economic benefits. Simulation runs are usually computationally difficult and it is not unusual for complex simulation models that they last for hours. However, decision making support, exploratory analysis and rapid adaptive calculations often require simplicity for understanding and explanation of representations of reality. Therefore, there are often constructed simpler approximations - models of simulation model - or metamodels which are used for studying of a computer simulations' behavior.

\section{Production process and its simulation}

Production process (Fig. 1) is a set of activities that require one or more types of inputs and create output which has value to the customer. It is a chain of operations in which material is changed to the product or order to the service for the customer. [7] Currently, a flexibility of manufacturing process is required, which enables a company to respond flexibly to market demands. Except for shortening a production cycle, there are also requests for a high productivity, flexibility of product mix, various production programs, stock reducing, increasing of machine utilization, quality improvement, rapid responses to emerging problems, delivery time shortening, etc. Therefore, planning and control system has an important role in an enterprise.

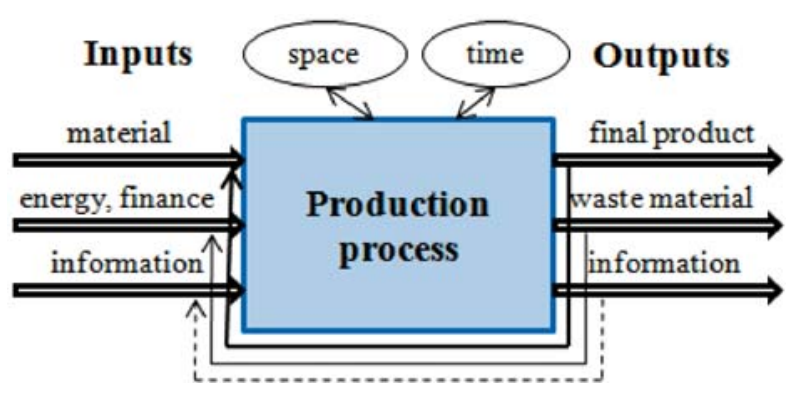

Fig. 1 Production process model

Qualitative information is provided by scheduling systems that enable forward simulation of possible options for future system's behavior and provide possibility of choice at the right moment. Such information is the base for an appropriate solution selection. Looking for optimal combination of input process factors needed for required changes execution is supported with simulation.

Simulation is a research method in which the experiments with a simulation model of manufacturing system are made on a com-

\footnotetext{
* Silvia Palajova, Milan Gregor

Department of Industrial Engineering, Faculty of Mechanical Engineering, University of Zilina, Slovakia, E-mail: silvia.palajova@fstroj.uniza.sk
} 
puter. It also ranks among statistical methods because it works on the same theoretical basis as methods of mathematical statistic. The analyst conducting simulation experiments selects a sample (statistical sample) that represents characteristics of whole examined file. Such sample is statistically analyzed and the results are applied to the whole file (population). Similarly, simulation also replaces the real system with its simulation model. This model includes only those characteristics of real system which the analyst is interested in. After evaluation of results the analyst makes conclusions about the whole real system, based on experiments with the model [8].

For practical applications of simulation optimization it is important that the optimization process is constrained within reasonable time limits and the efficiency of the optimization process is crucial One of the possible ways how to enhance effectiveness of simulation optimization and reduce the requirements of time-consuming simulation is to use computationally cheap metamodels [9].

\section{Simulation metamodelling}

Simulation metamodel [2] is a model of simulation model and it explains the fundamental nature of the system's input-output relationships through simple mathematical functions that enable to forecast output $Y$ for given input $X$ :

$$
Y=\underbrace{f(X, \beta)+\varepsilon}
$$

$Y=f(X, \beta)$ - regression function,

$Y$ - dependent variable,

$X$ - vector of values of input factors,

$\varepsilon-$ vector of random numbers.

This relationship is the regression model that expresses free (stochastic) dependence between explanatory variables $X$ and explaining variable $Y$. It means that for one particular combination of values of independent variables $X$ may $Y$ have various values. It is caused by an influence of random events $\varepsilon$. Thus, it is possible to assign count distribution of dependent variable $Y$, called conditional count distribution, which has its own mean and variance.

\subsection{Regression model}

According to the type of the regression function, regression models are divided into:

- linear models

$$
y_{i}=\beta_{0}+\beta_{1} x_{i 1}+\beta_{2} x_{i 2}+\ldots+\beta_{k} x_{i k}+\varepsilon_{i}
$$

with linear regression function

$$
\eta_{i}=\beta_{0}+\beta_{1} x_{i 1}+\beta_{2} x_{i 2}+\ldots+\beta_{k} x_{i k}
$$

where $\beta_{0}, \beta_{1}, \ldots, \beta_{k}$ are model parameters, $x_{i j}$ - i value $(i=1,2, \ldots, n)$ of explanatory variable $X_{j}(j=1,2, \ldots, k)$.

If the linear regression model has one independent variable $(k=1)$, the regression function is a line. If there are two explanatory variables $(k=2)$, the regression function is a plane. For more explanatory variables $(k \geq 2)$, we are talking about hyperplane. Linear models are most commonly used.

- linear-able models that may be adjusted to the linear form by simple transformation:

a) non-linear models in the independent variables but linear in parameters,

b) non-linear models in the parameters,

- non-linear (no linear-able) models which cannot easily be transformed into a linear form. Their regression function is e.g. modified exponential curve:

$$
\eta_{i}=\beta_{0}+\beta_{1} \cdot \beta_{2}^{x_{i}},
$$

logistic curve (S-curve that is symmetrical about an inflection point):

$$
\eta_{i}=\frac{\beta_{0}}{1+\beta_{1} \cdot \beta_{2}^{x_{i}}},
$$

Gomperz's curve (S-curve that is asymmetrical about the inflection point):

$$
\eta_{i}=\varepsilon^{\beta_{0}+\beta_{1} \cdot \beta_{2}^{x_{i}}},
$$

or curve with such recipe:

$$
\eta_{i}=\sqrt{\beta_{0}+\beta_{1} x_{i 1}+\beta_{2} x_{i 2}}+\beta_{3} x_{i 3},
$$

Regression analysis of nonlinear models uses different methods and procedures than linear models [10].

The aim purposes of regression and correlation analysis are [11]:

1. to verify the existence of dependence of explaining variable on considered explanatory variables,

2. evaluation of individual influence of explanatory variables on explaining variable,

3. forecasting explaining variable values for desired combinations of values of explanatory variables.

\subsection{Metamodel making process}

The metamodel creation (Fig. 2) begins with a simulation model which is preceded by defining the problem, defining the scope of input variables, the draft of the plan of experiments. After construction of a computer simulation model, its validation and verification is made, so logical structure of the model with respect to the real system is proved. Then a predefined number of replications for different input values is executed with the simulation model. In order to continue in metamodel making process we must be sure that data are sufficiently independent. 


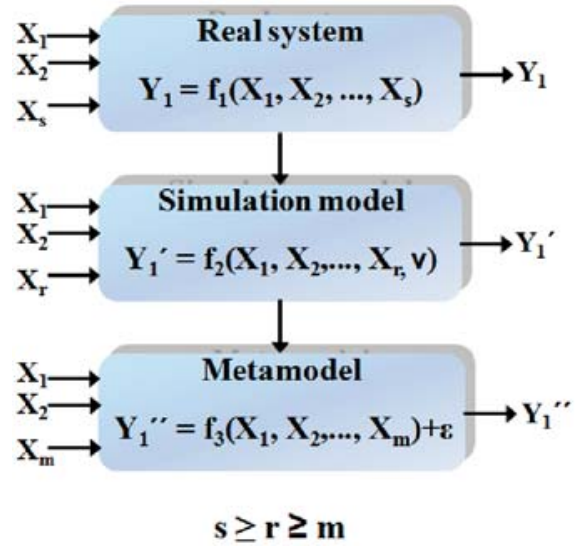

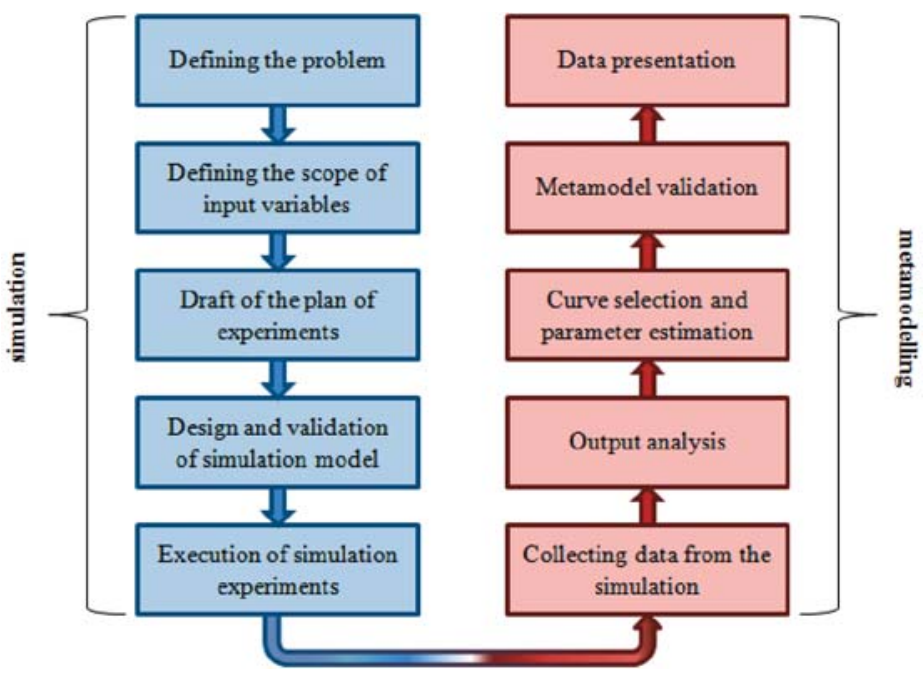

Fig. 2 Metamodel making process
In the next step, output data from the simulation are collected. In order to simplify the metamodel it is possible to combine some of the entries and remove those which have proved to be needless. These results are used for deriving a statistical model in the form of regression.

The heart of metamodelling is to determine a vector $\beta=\left(\beta_{1}\right.$, $\left.\beta_{2}, \ldots, \beta_{p}\right)^{T}$ which is a set of coefficients that determine regression function. Some of these coefficients, perhaps all, are unknown and they have to be estimated by adjusting the regression function to observed simulation results. The method of least squares is the most common method for estimation of regression functions. It is used for calculation of functions, providing its estimation is linear in parameters or it can be achieved by simple transformation. In fact, we do not know the type of regression function or its parameters $\beta$. Therefore, on the basis of empirical data, we must properly capture the character of dependence between dependent variable and independent variables (so choose an appropriate type of regression function) and estimate function parameters. The method determines the $Y_{j}^{\prime}$ estimation of the regression function

$$
Y_{j}^{\prime}=f\left(x_{1 j}, x_{2 j}, \ldots, x_{k j} ; b_{0}, b_{1}, \ldots, b_{p}\right)
$$

where coefficients $b_{0}, b_{1}, \ldots, b_{p}$ are estimations of unknown parameters $\beta_{0}, \beta_{1}, \ldots, \beta_{p}$. The difference between empirical and theoretical value of the dependent variable is the random error [1]:

$$
e_{j}=Y_{j}-Y_{j}^{\prime}
$$

If the random errors apply

$$
\begin{aligned}
& E\left(e_{j}\right)=0 \\
& \mathrm{D}\left(e_{j}\right)=E\left(e_{j}^{2}\right)=\sigma^{2} \\
& E\left(e_{j 1}, e_{j 2}\right)=0 \text { for each } j_{1} \neq j_{2}
\end{aligned}
$$

coefficients $b_{0}, b_{1}, \ldots, b_{p}$ may be considered as the best estimates of parameters $\beta_{0}, \beta_{1}, \ldots, \beta_{p}$. (10) shows that random errors have to have a normal distribution with zero mean value (in the case of sufficiently large statistical file) and constant variance (not necessarily, the variance may also vary proportionally). They are independent from $X i$ and should be mutually independent in pairs.

Least-squares condition is that sum of squares of random errors (residual deviations) of dependent variable has to be minimal:

$$
F\left(b_{0}, b_{1}, \text { É }, b_{p}\right)=\sum_{j=1}^{n}\left(Y_{j}-Y_{j}^{\prime}\right)^{2}=\min .
$$

Coefficients $b_{0}, b_{1}, \ldots, b_{p}$ have to suit this requirement. If also a specific type of function (8) is known, it can be inducted into relationship (11) and look for a minimum of the function. The result of the partial derivations of the function $F\left(b_{0}, b_{1}, \ldots, b_{p}\right)$ is then set up to be equal to zero:

$$
i=0,1,2, \ldots, p
$$

and the unknown coefficients $b_{0}, b_{1}, \ldots, b_{p}$ are calculated by solving of a system of $(p+1)$ equations about $(p+1)$ unknown quantities. A main disadvantage of the method of least squares is its sensitivity to extreme values and the only one outlier can change a direction of the regression line. Therefore the regression analysis should always start with looking over $X-Y$ chart [10].

The values of the vector $\beta$ are used for creating of curves that describe the metamodel. In order to check a suitability of the metamodel for intended purposes, validation of the metamodel (by comparison of metamodel with simulation output data using mathematical statistics) is done. The graphical representation of metamodel's inputs - outputs relationships provides a simple presentation of expected system behavior, often known as the approximate control. 


\section{Conclusion}

Mathematical models are usually used for systems' experimentation. They represent a system in terms of logical and quantitative relationships that can be manipulated and changed in order to find out how the model responds, so as the real system would respond. Many systems are quite difficult for application of analytical solutions on them. There can also occur uncertain conditions with insufficient information and without possibility of solution demarcation In such a case, the model must be studied by means of simulation. As these models are too complex, their appropriate approximations - called metamodels - are constructed. They relate output simulation data to metamodel's inputs and take into account the random effects occurring in the process. Simulation metamodelling is an appropriate tool for managing and optimization of complex manufacturing systems.

Simulation metamodelling is an appropriate managing and optimizing tool for complex manufacturing systems. The research work [6] was done at the Department of Industrial Engineering of the University of Żilina and it deals with the system analysis of input factors influence on the performance of manufacturing system.
This approach uses computer simulation and metamodelling principles, and proposed methods were verified in practical conditions. Other publications focus on metamodelling as a support tool in the frame of Digital factory [4], as a practical approach for a statistical summary of simulation results [3], [5], or as a support tool for designing and testing the control principles in production [12]. Theoretical assumptions and developments were validated on the chosen production system.

The authors' future research is focused on the design and verification of algorithm for approximate production control using simulation metamodelling. A detailed procedure for the metamodel's design will be proposed and evolutionary methods, namely neural networks, will be used in order to improve optimization. A system for automatic generating of metamodel will also be designed and proposed solution will be verified in the framework of the ZIMS research project (Zilina Intelligent Manufacturing System).

\section{Acknowledgement:}

This paper is the part of research supported by: ASFEU No. NFP26220220100.

\section{References}

[1] BAKYTOVA, H., UGRON, M., KONTSEKOVA, O.: ABC of Statistics. Bratislava : ALFA, editorship of technical and economic literature, 1975, p. 390.

[2] BARTON, R. R.: Metamodels for Simulation Input-output Relations. In Proc. Of $24^{\text {th }}$ Winter Simulation Conference, New York : IEEE, 1992. ISBN 0-7803-0798-4, p. 289-299.

[3] GREGOR, M.: Metamodel Design for Modelling and Simulation of Continuous Manufacturing Systems, 2005.

[4] GREGOR, M., HROMADA, J., MATUSZEK, J.: Digital Factory Supported by Simulation and Metamodelling, Inzinieria produkcji, 2008.

[5] GREGOR, M., STEFANIK, A., HROMADA, J.: Lean Manufacturing Systems Optimisation Supported by Metamodelling, 2008.

[6] HROMADA, J. Simulation of Manufacturing Systems, thesis, Zilina, 2004.

[7] KORBEL, P. Production Systems, study materials, Zilina : KPI, 2002.

[8] KOSTURIAK, J. et al.: Designing of Production Systems for the $21^{\text {st }}$ century. Zilina : EDIS ZU, 2000, p. 398. ISBN 80-7100-553-3.

[9] PERSSON, A., GRIMM, H., NG, A.: Simulation-based Optimisation Using Local Search and Neural Network Metamodels. [online] [cit.2010-06-10]. Available on the internet: http://www.his.se/

[10] RIMARCIK, M.: Statistics for Practice. Editorship Marian Rimarcik, 2007, p. 200, ISBN 978-80-969813-1-1.

[11] SOLTES, E. Regression and Correlation Analysis with Applications. Bratislava : Iura Edition, spol. s r. o., 2008, p. 288, ISBN 97880-8078-163-7.

[12] SKORIK, P., STEFANIK, A.: Emulation Environment - Reliable Approach for Designing and Testing of Management Principles in Production Based on Simulation, Optimization and Metamodelling, InvEnt 2010. 